
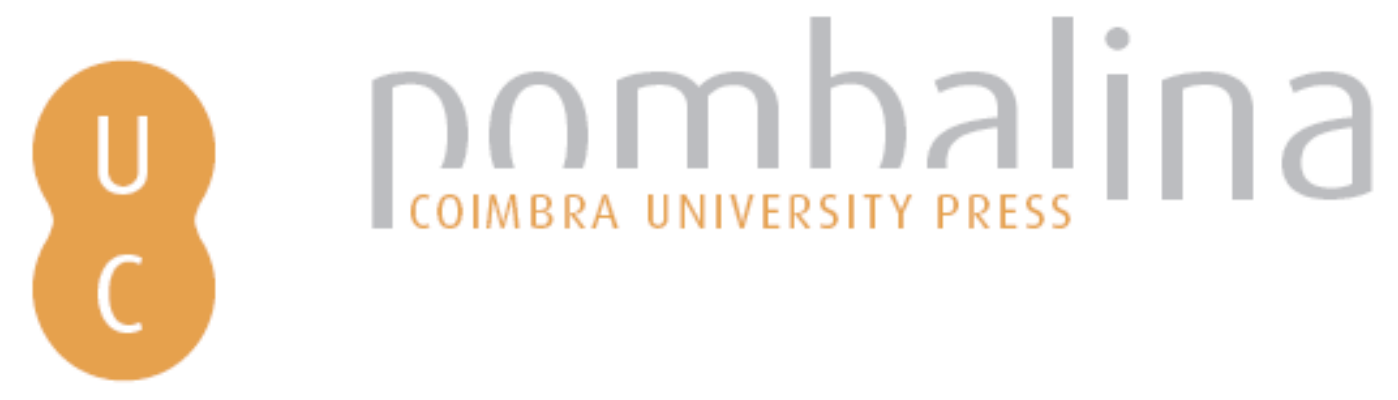

\title{
Virtus romana et taedium uitae: remarques sur l'évolution des mentalités et de la morale à l'époque de Martial
}

\author{
Autor(es): $\quad$ Robert, Jean-Noel \\ Instituto de Estudos Clássicos, Centro de Estudos Clássicos e \\ Publicado por: Humanísticos; Departamento de Estudos Clássicos, Centro de Estudos \\ Clássicos

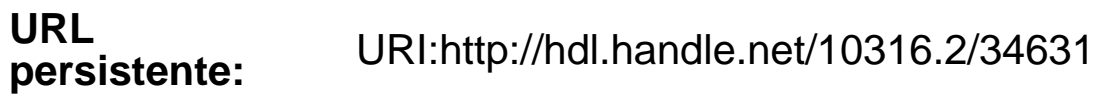 \\ DOI: DOl:http://dx.doi.org/10.14195/978-989-26-0901-0_5 \\ Accessed : $\quad$ 26-Apr-2023 16:08:45
}

A navegação consulta e descarregamento dos títulos inseridos nas Bibliotecas Digitais UC Digitalis, UC Pombalina e UC Impactum, pressupõem a aceitação plena e sem reservas dos Termos e Condições de Uso destas Bibliotecas Digitais, disponíveis em https://digitalis.uc.pt/pt-pt/termos.

Conforme exposto nos referidos Termos e Condições de Uso, o descarregamento de títulos de acesso restrito requer uma licença válida de autorização devendo o utilizador aceder ao(s) documento(s) a partir de um endereço de IP da instituição detentora da supramencionada licença.

Ao utilizador é apenas permitido o descarregamento para uso pessoal, pelo que o emprego do(s) título(s) descarregado(s) para outro fim, designadamente comercial, carece de autorização do respetivo autor ou editor da obra.

Na medida em que todas as obras da UC Digitalis se encontram protegidas pelo Código do Direito de Autor e Direitos Conexos e demais legislação aplicável, toda a cópia, parcial ou total, deste documento, nos casos em que é legalmente admitida, deverá conter ou fazer-se acompanhar por este aviso.

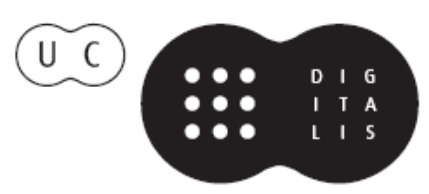




\section{Toto notus in orbe Martialis}

\section{Celebração de Marcial \\ 1900 anos após a sua morte}

Coordenação

Cristina de Sousa Pimentel

Delfim F. Leão

José Luís L. Brandão

Instituto de Estudos Clássicos

Centro de Estudos Clássicos e Humanísticos

UNIVERSIDADE DE COIMBRA
Departamentō de Estudos Clássicos Centro de Estudos Clássicos UNIVERSIDADE DE LISBOA 


\section{VIRTVS ROMANA ET TAEDIVM VITAE REMARQUES SUR L'EVOLUTION DES MENTALITES ET DE LA MORALE A L'EPOQUE DE MARTIAL}

JEAN-NOËL ROBERT

Faculté Libre de Paris

Résumé: L'époque de Martial fut le témoin d'une évolution dans les mentalités due à des facteurs politiques, économiques et sociaux, et d'une transformation de la morale républicaine. Rome adapte aux circonstances nouvelles les anciennes vertus qui ont fait sa grandeur depuis ce que l'on peut appeler «le miracle romain" (au IIe siècle avant notre ère). Elle s'est toujours sentie investie d'une mission, celle de gouverner le monde. Son vaste empire doit son équilibre à l'ouverture de sa civilisation à toutes les cultures de l'humanité et à l'instauration d'une société d'intégration et de tolérance. Le système romain des valeurs compte pour beaucoup dans la réussite de cette entreprise de paix unique dans l'histoire, et notamment la fides.

Comment alors justifier le taedium uitae et l'intempérance dénoncée par Sénèque et dépeinte par Martial et Juvénal? L'individualisme (sibi uiuere), la prééminence de l'argent expliquent en partie la réalité de la crise morale, mais la lecture de Pline ou d'Apulée permet d'entrevoir les nouvelles valeurs qui régissent les rapports entre les hommes. De cette crise profonde et salutaire se dégage un humanisme universel qui constitue le ferment culturel de notre unité européenne moderne.

Tacite note qu'à l'époque de Néron, «les mœurs traditionnelles» sont bouleversées, que la jeunesse a perdu «les qualités ancestrales» et que, lors des nuits de fête, «les gens les plus abominables...os[ent] dans 
les ténèbres ce qu'ils [ont] désiré pendant le jour ${ }^{1} \gg$. Martial et Juvénal sont les témoins privilégiés d'une crise morale en train de se dénouer, et d'une évolution profonde de la mentalité romaine qu'il faut tenter d'analyser pour comprendre comment certains philosophes, à partir de Sénèque, ont posé les fondements de notre monde moderne et de la morale dite judéo-chrétienne au moins autant que les textes sacrés.

Mais une lecture par trop négative des moralistes et des satiristes ne laisserait à celui qui les étudie que la vision d'une Rome décadente et débauchée. Tel n'est pas le cas, car les chroniqueurs n'aiment retenir que l'excès, c'est-à-dire l'exceptionnel. Au premier siècle de notre ère, la société change parce que le cadre politique s'est modifié, parce que l'étendue de l'empire atteint bientôt son maximum, parce que la Rome impériale n'a plus grand chose de commun avec la cité de Caton au lendemain de la seconde guerre punique. C'est dans ce contexte qu'il convient de resituer la dégradation morale comme un prélude à une mutation vers la situation nouvelle.

En réalité, Rome est à l'apogée de sa force et se transforme avant de poser les fondements culturels de notre Europe moderne. Elle doit sa réussite à la conscience qu'elle acquiert de sa situation originale dans l'Antiquité: elle associe l'humilité, la déférence, l'admiration même qu'elle éprouve vis à vis de la culture grecque, plus ancienne, dont elle $\mathrm{s}^{\prime}$ est nourrie, et le devoir qu'elle assume de forger une culture universelle au monde qu'elle domine et dont elle se sent responsable.

Cette science du compromis, cet art de la transmission, dans l'abnégation de celui qui sait avoir toujours quelque chose à apprendre, est parfaitement exprimé dans ces quelques lignes du livre de Rémi Brague $^{2}$, qu'il faut citer: «Est romain, quiconque se sent pris entre quelque chose comme un hellénisme et quelque chose comme une barbarie. Etre romain, c'est avoir en amont de soi un classicisme à imiter, et en aval de soi une barbarie à soumettre. Non pas comme si l'on était un intermédiaire neutre, un simple truchement lui-même étranger à ce qu'il fait communiquer, mais en sachant que l'on est soi-même la scène sur laquelle tout se déroule, en se sachant soi-même tendu entre un classicisme à assimiler et une barbarie intérieure. Etre romain, c'est se perce-

1 Tacite, Annales, 14. 20, 4-5.

2 Rémi BRAGUE, Europe, la voie romaine (Paris, Criterion, 1992). 
voir comme grec par rapport à ce qui est barbare, mais tout aussi bien comme barbare par rapport à ce qui est grec. C'est savoir que ce que l'on transmet, on ne le tient pas de soi-même, et qu'on ne le possède qu'à peine, de façon fragile et provisoire. La culture romaine est ainsi essentiellement passage.» Rome n'a peut-être pas inventé grand chose, mais elle a su transmettre et accorder sous une même patine les cultures qui, autrement, se seraient, au mieux ignorées, au pire, combattues.

Tel est ce que j'appelle le miracle romain, et dont la plus belle manifestation se situe au II siècle avant notre ère, sous la République triomphante, lorsque, en un demi siècle, Rome a su assimiler la culture grecque sans perdre son identité traditionnelle, mais au contraire en faisant son miel d'une culture plus riche et plus ancienne que la sienne dont elle a gardé ce qui pouvait la vivifier elle-même ${ }^{3}$.

Il faut dire qu'à cette époque glorieuse, Rome n'en est pas à ses débuts en matière d'acculturation. Il s'agit d'un trait fondamental de la mentalité romaine, et les Romains y ont été confrontés dès avant la fondation de leur Ville. Influences grecques et étrusques, association avec les Sabins, membre de la Ligue latine dont elle prend progressivement la tête, conquête de l'Italie (et des Grecs du sud), confrontation avec Carthage..., l'œuvre de Rome, contrairement à ce que l'on croit, n'est pas une oeuvre de guerre, mais de paix. Il s'agit à chaque fois de romaniser, c'est-à-dire d'offrir la protection de Rome aux vaincus et de les assimiler. C'est ainsi que la petite cité du Latium, une parmi tant d'autres, vit sa vocation à s'étendre jusqu'aux confins du monde. Vocation, ou plutôt mission, telle que définie par Jupiter au glorieux ancêtre, Enée, selon Virgile: «d'autres... façonneront avec plus de souplesse un bronze qui semblera respirer; ils tireront du marbre des visages plein de vie; ils plaideront mieux leurs causes; ils décriront au compas les mouvements célestes et diront le lever des constellations: toi, Romain, souviens-toi de régir les peuples sous ton empire. Ce sont là tes talents, ainsi que d'imposer des conditions de paix, d'épargner les vaincus et de soumettre les insolents ${ }^{4} . »$ Enée n'est pas un héros épique ordinaire, comme ceux $\mathrm{d}^{\prime}$ Homère qui combattent pour assouvir une vengeance personnelle, il est investi d'une mission divine, et confère à Rome sa vocation universelle: gouverner le monde.

${ }^{3}$ Cf. Jean-Noël Robert, Caton ou le citoyen (Paris, Les belles Lettres, 2002).

${ }^{4}$ Virgile, Enéide 6. 847-853. 
A l'origine de cette belle assurance qui conforte le Romain dans son action, il y a le sentiment de se sentir physiquement et humainement au centre du monde. Nombre de témoignages littéraires corroborent cette certitude, jusqu'à l'architecte Vitruve qui note: «on peut dire que les Romains, placés entre le nord et le midi, possèdent tout ce qu'il y a de meilleur entre ces deux extrémités du monde; car ils jouissent d'un climat tempéré, et par prudence ils triomphent de la force des barbares: de même que, par leur valeur, ils déjouent l'astuce et l'adresse des peuples méridionaux. Le ciel a donc placé la capitale du peuple romain dans une région merveilleusement tempérée, pour qu'elle fût capable de commander à toute la terre ${ }^{5}$.»

Ces propos, dans la bouche de l'architecte, montrent bien tout le symbolisme de la ville. Rome, c'est d'abord l'image de la Ville, et l'imaginaire urbain se calque sur l'image du monde. La ville, comme l'empire, est défini par des frontières qui sont, pour un Romain, le symbole de sa sécurité. Romulus a fait descendre sur la terre le templum de Jupiter et tracé le sillon sacré que nul ne pouvait franchir, comme Rémus, sans mériter la mort salvatrice pour la ville. Les frontières justifient le mythe de Rome, projet divin, assurant l'intégrité de la ville, donc celle de l'empire. Les conquêtes ne font que poser des limites secondes qui élargissent en cercles concentriques le cercle de la cité. Rome s'impose au monde par cercles concentriques et se pense comme le centre de l'univers.

Mieux encore, la cité elle-même se pense comme l'orbis terrarum. "L'espace de Rome est à la fois celui de la ville et du monde», dit Ovidé. L'ambition de Rome est d'étendre aux limites de l'univers ce modèle romain de concorde et de paix manifesté dans l'enceinte sacrée de la ville. L'édit de Caracalla ne fait que confirmer l'aboutissement du projet unificateur de Rome. Rome est la garante de l'ordre et de la cohérence, et sa structure se retrouve dans toutes les villes de l'empire. L'espace y est organisé en lieux de pouvoir, de la politique, de la justice, de l'activité économique, des loisirs... Rome est unique, et le modèle s'en reproduit. Roma caput mundi est-il écrit sur une pierre du Circus Flaminius? ${ }^{7}$. Un

${ }^{5}$ Vitruve, De l'architecture 6. 1.

${ }^{6}$ Ovide, Fastes 2. 683-684.

7 Tel était déjà la signification du prodige de la tête trouvée sur le Capitole (Tite-Live, 1. 55, 1-6). L'idée de cette vocation à diriger le monde s'est affirmée 
espace urbain apaisé au sein duquel les armes sont bannies, et rejetées également aux frontières de l'empire, où le pain et les jeux devront garder l'illusion de vivre ensemble et en paix.

Rome se situe donc au centre de tous les équilibres, au cœur des cultures de l'humanité. «La nature a posé ici tout ce qui était partout», note Properce, comme avec lui, dans des termes voisins, Virgile et Horace. Cela suppose d'intégrer à l'utopie tout ce qui lui est étranger, l'autre, le barbare. Ce qui va sans peine: depuis l'enlèvement des Sabines, la société romaine est faite de métissage, d'intégration et de tolérance. Outre la tolérance religieuse bien connue qui fait accueillir et respecter à Rome toutes les religions de l'empire, il faut insister sur la tolérance sociale. Il n'existe à Rome aucun racisme en tant que tel, tout au plus des manifestations de xénophobie. Mais à y regarder de plus près, on s'aperçoit que le Romain ne développe pas une image de l'étranger en tant que tel. Les défauts stéréotypés que le Romain perçoit chez l'étranger sont en réalité des attitudes qui s'opposent aux qualités qui définissent un bon Romain. La superstition des uns accuse un manque de pietas, la mollesse des orientaux, un manque de uirtus, la ruse et le manque de loyauté des Grecs ou des Carthaginois, un défaut de fides, l'indiscipline des Gaulois, un manque de maîtrise de soi et une légèreté trahissant l'absence de grauitas. Ces défauts, qui ne sont rien d'autre que l'envers des qualités $d^{\prime}$ un bon romain, n'entravent en rien la volonté d'assimilation qui fait se demander à un Denys d'Halicarnasse comment, au fil de ses conquêtes, Rome ne s'est pas «entièrement barbarisée ${ }^{8}$ ». Attitude complètement incompréhensible à un roi oriental comme Mithridate qui

pendant le dernier siècle de la République. Cicéron l'évoque dès -81 pour parler des pouvoirs de Sylla (imperium orbis terrarum, dans la Rhétorique à Hérenius 4. 13). Pompée, lors de son triomphe de -61 fait graver dans la pierre qu'il a fait reculer «l'Empire romain jusqu'aux limites de la terre» (Diodore de Sicile, 40. 4), Cornélius Népos reprend la formule pour stigmatiser les ambitions d'Antoine et d'Octave (Vie d'Atticus 20.5), et Auguste ouvre ses Res gestae en rappelant qu'il a «soumis l'univers à l'Empire du peuple romain.» Il fut d'ailleurs le premier à matérialiser ce pouvoir dans la ville, tant par l'organisation symbolique de son forum que par la conception de l'horologium.

${ }^{8}$ Denys d'Halicarnasse, Antiquités romaines 1. 90. 
traite Rome de «dépotoir d'immigrants ${ }^{9}$ ». Et il faut bien reconnaître que le cosmopolitisme qui règne à Rome sous l'Empire fait sans doute craindre à certains, comme Juvénal, la perte d'une identité romaine, mais la réalité condensée de la capitale ne correspond pas forcément à celle des provinces.

En fait, la réussite d'une intégration qui a pacifié un aussi grand empire sur une aussi longue période - un fait unique dans l'histoire de l'Antiquité - vient d'abord d'une prise de conscience, celle d'une responsabilité envers les vaincus, dont parle déjà un Cicéron. Les vaincus sont respectés dans leurs droits. Ils conservent leur langue, leurs coutumes, leurs lois et jouissent de la protection de Rome. La paix assure leur prospérité. La romanité devient un véritable marché commun. La colonisation romaine est une dimension décisive dans l'essor de la latinité. L'armée est elle-même une machine à romaniser. Les empereurs eux-mêmes sont issus d'horizons de plus en plus lointains: les Julio-Claudiens étaient membres des plus anciennes familles de Rome, les Flaviens descendent de familles de notables italiens, les Antonins peuvent avoir des ascendants issus d'Espagne ou de Gaule narbonnaise; Caracalla est fils d'un Africain et d'une Syrienne etc... Et certains ne parlent même pas le latin! A l'époque de l'Edit de Caracalla (212), descendants des vainqueurs et descendants des vaincus se confondent dans une "patrie romaine universelle» de soixante millions d'habitants ou plus. Chaque citoyen de l'empire n'a, à l'évidence, qu'une seule citoyenneté officielle, celle de Rome, mais celle-ci reste souvent abstraite, et il n'abandonne jamais le sentiment d'appartenir à sa "petite patrie». De Rome, il obtient un statut, des avantages importants (droit de mariage, de commerce etc...) et droit de justice, comme Paul de Tarse qui, arrêté, sera traité avec égard lorsqu'il affirmera sa citoyenneté romaine et, condamné à mort, sera décapité au lieu d'être jeté aux lions. Le principal bénéfice de ces Romains de l'Empire, c'est de bénéficier d'un droit très élaboré et de jouir d'une dignitas qui est la signature d'un monde civilisé.

A propos de cette citoyenneté étendue à tous les habitants de l'Empire en 212, il faut souligner l'aspect paradoxal de cet édit: d'une part, il ne fait que prolonger en la concrétisant dans la loi une évolution dont l'origine remonte à la Guerre sociale de -89; mais d'autre part, il représente un acte révolutionnaire en ce qu'il généralise à toute une

${ }^{9}$ Le mot est rapporté par Justin, 38. 7.1. 
population cosmopolite une situation qui était réservée à une élite romaine. Cet état de fait ne signifie pas que la société ait cessé d'être inégalitaire, mais il représente l'acte suprême d'une politique fondée sur la conscience responsable de reconnaître une forme théorique d'égalité devant la loi de chacun des hommes qui composent un empire. C'est le meilleur facteur de paix. N'oublions pas que les lézardes survenues dans l'empire romain et son ébranlement ne viennent pas de l'intérieur, mais de l'extérieur, des barbares, souvent d'ailleurs envieux des privilèges accordés par l'empereur.

Mais ce qui a d'abord assuré le succès de Rome, ce sont les qualités humaines organisées en un système de valeurs à la fois cohérent et complexe. Ces valeurs expriment parfaitement l'humanisme romain et sont réunies dans ce qui constitue le cœur de Rome, à savoir l'homme social, politique et civique; autrement dit le citoyen.

Il faut ici, une fois encore, redire combien il serait dangereux, parce que le mot «citoyenneté» revêt pour nous une importance fondamentale dans notre idéal de démocratie, de faire l'amalgame entre son sens dans l'Antiquité, à Rome, et celui d'aujourd'hui. La citoyenneté antique ne se fonde pas sur les mêmes rapports civiques que la nôtre.

Avant de parler des valeurs qui définissent le citoyen romain, il faut sans doute passer par un bref rappel de ce que le mot lui-même signifie.

En latin, ciuis fait couple avec hostis (l'étranger à la cité). Ciuis est de la même racine que le grec keimai qui signifie habiter (et d'où dérive notre français «hameau»). Le citoyen est celui qui, à l'époque royale, habite en famille (au sens large), et ce sont ces familles qui constituent une structure plus large, appelée citoyenneté, concrétisée par la réalité de la cité. Le mot ciuitas désigne à la fois la cité et la citoyenneté.

A Rome cohabitent donc ceux qui y habitent, les citoyens, et les autres, les esclaves, qui peuvent être affranchis et ainsi devenir libres, leurs enfants jouissant de la complète citoyenneté, selon un processus d'intégration particulièrement développé. Rappelons par exemple le cas d'Horace, proche du pouvoir, dont pourtant le père était un affranchi, et le grand-père un esclave.

Cependant, il ne faut pas croire que l'on naît citoyen de droit. Un fils de citoyen doit d'abord être reconnu par son père et inscrit sur les listes de la cité. Là intervient le destin: un enfant non reconnu est abandonné ou éliminé, peut être récupéré par une autre famille qui en fera son 
esclave. De la même façon, un citoyen reconnu peut être déchu de ses droits s'il ne s'est pas inscrit sur les listes du censeur, s'il a failli à ses devoirs ou s'il a été kidnappé par des pirates qui le revendent comme esclave à l'autre bout de la Méditerranée, comme cela manqua d'arriver à César lui-même.

Ce qui compte, pour un père, c'est d'avoir un héritier en bonne santé qui puisse servir l'honneur de son nom et assurer sa succession. Peu importe alors qu'il soit de son sang; il peut adopter le fils d'un autre citoyen et en faire le sien propre. Le fils aîné de Paul Emile fut adopté par un Fabius, et le suivant par le fils de Scipion l'Africain, devenant le célèbre Scipion Emilien.

D'ailleurs la réussite du système romain repose sur sa solide armature juridique. Rome a toujours veillé à un parfait équilibre entre les droits et les devoirs. Le citoyen a des devoirs importants: s'inscrire sur les listes de la cité, participer à la vie politique et servir sa patrie en payant ses impôts et en effectuant des campagnes militaires lorsqu'il y a menace venue de l'extérieur. Il doit aussi respecter la loi commune qui est, dès l'origine, définie comme celle des ancêtres (mos maiorum) et inscrite dans les Tables (dite Loi des XII Tables). Cela met en évidence une caractéristique essentielle du citoyen romain: il n'existe pas en tant qu'individu. Il appartient à plusieurs groupes au sein desquels il remplit ses obligations. Seule compte la cité, cette communauté dont la force ne vient que de l'union et de l'abnégation de ses membres. Ainsi, au sein de la cité, le citoyen appartient à sa famille (la gens), celle qui l'a reconnu, puis, à sa majorité, devient membre d'une tribu (circonscription géographique) et d'une centurie, suivant sa fortune, qui lui permettent d'exercer son métier de citoyen: participer aux assemblées où se votent les lois et s'élisent les magistrats. Du reste, il n'exprime pas sa voix individuellement, mais c'est à chaque fois l'ensemble de la tribu, ou celui de la centurie qui exprime une même voix. Ainsi le citoyen n'est-il rien en dehors de la collectivitétio.

On peut certes noter l'injustice du système électoral qui, depuis la réforme dite de Servius repose avant tout sur la fortune du citoyen, mais

10 Le fait que le citoyen n'existe qu'au sein d'une collectivité est particulièrement patent sous la République dans le fonctionnement du système électoral. Cf. Claude Nicolet, Le métier de citoyen dans la Rome républicaine (Paris, Gallimard, 1976). 
il faut noter que c'est aux plus riches que reviennent les obligations les plus lourdes, et notamment celle de défendre la cité, du moins jusqu'à la réforme de Marius, parce que l'on pensait que, pour bien défendre sa terre, il faut en posséder soi-même une partie. Il y a donc une mesure de justice dans la répartition des avantages et des charges.

En outre, le système clientéliste romain, fondé lui aussi sur la fortune, donne aux plus riches la charge d'entretenir les plus pauvres. A l'exception toutefois des plus démunis dont on ne peut rien attendre, car le système d'échange en vigueur suppose que le client a quelque chose à offrir à son patron en échange de sa protection et de la sportule ${ }^{11}$.

Cette protection des autres citoyens constitue l'un des principaux droits que chacun peut attendre de la collectivité. Il s'agit d'une des valeurs les plus remarquables et les plus caractéristiques de la République romaine. C'est la fides, c'est-à-dire le dévouement, le devoir de servir, la loyauté que tous doivent à chacun comme chacun la doit à sa patrie et à la collectivité. L'application du ius ciutle donne le droit à chacun de faire appel au peuple (prouocare ad populum), ce qui montre qu'à époque ancienne, les institutions civiques ne fonctionnaient pas encore bien et que l'on pouvait réparer une injustice en demandant la solidarité des citoyens envers soi. Ce lien avait une force religieuse car ce respect des lois et des règles sociales se complique toujours, à l'origine, d'un aspect religieux, d'une dimension mystique. Ce que disait très bien Cicéron: «par la loi, les hommes se trouvent associés aux dieux ${ }^{12}$.»

Le mot fides a donc à la fois un sens actif (la fides que je donne) et un sens passif (celle que je reçois). Il s'agit alors d'une protection accordée et reçue, que, par exemple, un homme condamné injustement peut implorer de l'assemblée de ses concitoyens pour une sorte de jugement en appel. Cette notion de «protection due» à un citoyen suppliant est un élément clé de la morale romaine archaïque qui n'existe pas de cette façon en Grèce. C'est elle qui a conduit au développement de clientèles privées à Rome et à l'intérieur de l'Italie comme dans les provinces de l'empire, formant ainsi l'ossature de l'empire romain. Ce lien qui existe entre citoyens est encore renforcé entre soldats (qui sont aussi des citoyens à

${ }^{11}$ Cf. notre contribution, dans ces mêmes Actes: «Société et cultus à l'époque de Martial».

${ }^{12}$ Cicéron, De officiis, 3. 29. Cf. la thèse de Gérard FreYBURger, Fides (Paris, Les Belles Lettres, 1986). 
l'origine) puisqu'ils sont liés entre eux par le sacramentum militiae. Il est fort probable qu'il y ait eu une cérémonie religieuse à la base de ce lien de fidélité entre citoyens, et peut-être même lors de la prise de la toge virile, dans une cérémonie d'accueil des nouveaux citoyens, parmi les différentes manifestations et sacrifices de ce jour des Liberalia. Les jeunes se rendaient au Capitole au temple de Jupiter, mais s'y trouvait aussi le temple de Fides. Non loin de ce temple, l'ara gentis Iuliae où était affichée la lex Vespasiani de ciuitate danda. Le terme de Quirites (d'où dérive la curie $<\mathrm{CO}^{-}$ uiria) évoque le rassemblement des hommes en organisation civile et politique. Dans l'Aulularia, Euclion dit qu'à une récente réunion de sa curie, on l'a entouré et on lui a serré la main, geste plus rare

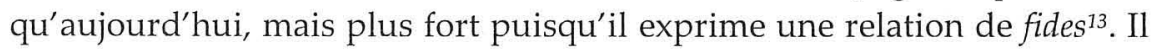
$\mathrm{n}^{\prime}$ est donc pas étonnant que ce lien de fides apparaisse comme «le fondement moral de l'ordre romain» (selon le mot de P. Boyancé14), véritable pierre angulaire du système social et idéologique de Rome.

Et l'on peut penser que c'est grâce à la force de son système moral que la Rome républicaine a pu s'imposer au reste du monde. A l'époque impériale, ce lien de fides horizontal entre citoyens s'estompe au profit d'un lien de fides vertical qui unit les sujets à l'empereur. La solidarité civique, et celle qui se manifeste naturellement entre êtres humains semblent s'être émoussées. Un citoyen n'éprouve plus le même sens du devoir envers ses concitoyens que sous la République. Mais la morale des Romains évolue vers d'autres valeurs qui sont bien différentes de celle des hommes qui étaient si orgueilleux de leur libertas.

La fides se trouve donc en tête des valeurs romaines, mais elle n'est pas la seule, et la réussite de Rome dépend de tout un système de valeurs cohérent et complexe, où se complètent pietas, uirtus, grauitas et d'autres comme autant de tuteurs inspirés de la tradition pour maintenir le Romain dans le chemin de sa gloire. Chacune d'elle est difficile à traduire tant elles ne recouvrent aucune de nos références morales actuelles.

Ainsi la pietas est-elle à la fois le respect et la soumission qu'un fils doit observer envers ses parents, un citoyen envers sa cité, un homme envers les dieux. La vertu par excellence, celle du «pieux Enée», celle dont Cicéron disait qu'elle était spécifiquement romaine et, suggérait-il,

${ }^{13}$ Plaute, Aulularia, v. 115-116

14 Pierre BoyAnCE, «Les Romains, peuple de la fides», in BAGB (Paris, 1964) 419-435. 
«c'est par cette sagesse exceptionnelle qui nous fait percevoir que la puissance des dieux règle et gouverne tout, que nous l'avons emporté sur tous les peuples et toutes les nations ${ }^{15}$.»

La fides et la pietas associées représentent la relation sécurisante, faite de confiance et de loyauté, qui garantit le lien entre les divers maillons de la société des citoyens, horizontalement entre un patron et son client, entre les membres d'une même famille, entre les citoyens, entre les vainqueurs et les soumis, mais aussi verticalement entre les hommes et les dieux.

Ce sont bien les deux qualités suprêmes, les deux «vertus cardinales» du Romain. Car le terme de vertu n'a pas non plus le sens qu'on lui accorde aujourd'hui. Certes il est souvent question de la célèbre uirtus romana, c'est-à-dire la qualité du uir à la fois à servir sa patrie et à pouvoir lui donner des fils qui poursuivront son action (ne dit-on pas à chaque naissance: «la cité s'est augmentée d'un fils»?), mais en réalité, il faudrait surtout parler des uirtutes. Car la uirtus est un ensemble de «vertus» qui toutes qualifient le courage, l'énergie du citoyen et son activité politique ainsi que sa dignitas (capacité à tenir son rang) et sa maiestas, sa vocation à dominer, la prééminence du magistrat élu sur ses concitoyens, la supériorité naturelle du Romain sur les autres peuples.

Comment alors, devant tant de grandeur, justifier la peinture des mœurs que nous livrent Martial et tant d'autres? Comment expliquer le pouvoir des sirènes corruptrices de l'intempérance qui, selon Sénèque, «détournent de la patrie, de la famille, des amis et des vertus ${ }^{16}$ »? A lire le philosophe, on comprend que les épigrammes et les satires stigmatisent sans tellement exagérer ce que le maître à penser de Lucilius nomme luimême «la perversion des mœurs»: "on cherche en tout et partout son plaisir. Il n'est point de vice qui demeure en ses limites. Le luxe incline à la cupidité. La notion de l'honnête est abolie. Il n'y a pas de honte là où sourit le profit. L'homme, chose sacrée pour l'homme, on l'égorge de nos jours par jeu et par passe-temps...»

${ }^{15}$ Cicéron, De haruspicum responsis 19.

${ }^{16}$ Sénèque, Epistulae 123. 10-12. Les Lettres à Lucilius 95 et 122 contiennent les principales critiques du philosophe sur les mœurs. 
La première cause de ce changement est politique. Le citoyen décrit par Caton n'existe plus. Il ne participe plus à la vie publique depuis que le pouvoir a été confisqué par un princeps qui n'est pas le primus inter pares dont rêvait Cicéron, mais un nouveau monarque. Le citoyen-acteur est devenu spectateur, et son arme, la parole, est confinée dans les écoles où elle continue de s'exercer, mais sur les sujets les moins politiques et les plus improbables. Il suffit de lire Sénèque le Rhéteur ou Quintilien pour s'en persuader. Le pouvoir, monopolisé, a déssaisi le citoyen de sa fonction. Désœuvré, celui-ci attend qu'on le distraie et qu'on le nourrisse. Panem et circenses. Or l'inertie se gave de sensationnel, et il en faut toujours plus pour combattre la lassitude née de la monotonie. C'est pourquoi Sénèque s'indigne de vivre parmi un peuple «ivre et vomissant», parce que «c'est maintenant décembre [c'est-à-dire les Saturnales] toute l'année».

La seconde cause est économique. L'évolution du pouvoir et le développement de l'empire ont favorisé la fortune mobilière et la puissance de l'argent. A lire un client comme Martial, on sent bien que tout se résume à cette différence: en avoir ou pas. Le degré de fortune définit du même coup le degré de reconnaissance sociale. Outre les riches, les patrons, il existe les «pauvres», ou ceux qui s'estiment tels parce qu'ils ne peuvent acquérir leur complète autonomie par rapport aux riches; ils sont donc les clients des riches. Et enfin les plus miséreux, les laissés pour compte, les sans abri, qui ne sont rien. La valeur morale d'un homme se mesure à l'aune de sa bourse: "si tu as un as, tu vaux un as», dit un personnage de Pétrone. Une grande partie de cette société vit donc, plus ou moins bien, aux dépens de la fraction aisée de la population. Le citoyen, devenu parasite, est d'abord un assisté.

Les effets sont divers, mais se réduisent tous à ce sentiment étrange de dégoût de la vie, ce taedium uitae, cet incommensurable ennui qui ronge l'âme romaine aux deux premiers siècles de notre ère, et à son antidote, la recherche frénétique du plaisir qui étourdit et procure l'oubli. Il s'agit, pour le citoyen, de s'abandonner aux vains artifices de la superficialité. Sénèque reproche à la plupart de n'avoir pour conviction que le credo des hédonistes: «manger, boire, jouir de son bien, c'est cela vivre...» Ainsi pense-t-on tromper la mort qui guette et qui effraie. Le plaisir comme masque de la détresse. Le citoyen ne pense plus, il s'abandonne à l'illusion du spectacle. Spectacle du corps, aux thermes, où les regards se teintent d'érotisme, spectacle de la recitatio, où l'on se rend davantage 
pour être vu que pour entendre un jeune auteur, à moins qu'on ne cherche à y glaner quelque bon mot qu'on ne manquera pas de faire sien pour éblouir ${ }^{17}$, spectacle de la mort, à l'amphithéâtre, à l'heure des exécutions de midi, pour s'y décharger de sa haine et de son agressivité. Les passions s'y déchaînent dans leur agressivité crue. Et les femmes ne sont pas épargnées, et «celle qui foule du pied le pavé malpropre ne vaut pas mieux que celle qui se fait porter sur les épaules de ses longs Syriens», avertit Juvénal18. Regardez Ogulnia, qui n'est pas riche, mais dilapide l'héritage paternel «jusqu'au dernier vase», ou Eppia qui quitte mari, enfants, sœur, maison... pour suivre "Petit Serge», un homme disgracieux et nauséabond (oui, mais «c'était un gladiateur»!), ou Laevina, jadis vertueuse, qui préfère délaisser son mari pour suivre un jeune amant, ou Vistilia, ou... Pour ne rien dire de Messaline. L'épisode de la matrone $\mathrm{d}^{\prime}$ Ephèse, dans le roman de Pétrone ${ }^{19}$, paraîtrait presque trop sage.

Une des principales manifestations de cette évolution de la mentalité pourrait se résumer dans une formule dont Plaute, déjà, montrait la nocivité pour la société romaine: le sibi uiuere, autrement dit l'instillation dans les mœurs de l'individualisme et de l'égoïsme. Plaute l'avait mis en scène dans un personnage-repoussoir, naturellement d'origine grecque, Périplectomène, le soldat fanfaron. Périplectomène concentre sur lui tous les défauts dont les Romains accablaient alors les Grecs ${ }^{20}$ : hautains, paresseux, arrogants..., mais surtout égoïstes et indifférents à leurs concitoyens. Rome, au contraire, tirait sa force de sa cohésion sociale et du fort sentiment de collectivité qui unissait les citoyens. Un visionnaire comme Caton avait bien compris tout le danger d'une désagrégation sociale par le développement de l'individualisme. Deux siècles et demi plus tard, le mal est fait, et les uirtutes romaines y ont mal résisté.

Le développement de l'individualisme a privé le citoyen du soutien que procure le sentiment d'être une part active d'un tout. Et, en même temps, l'ancienne religion n'est pas en mesure de répondre à des attentes personnelles. En s'isolant, et en se réfugiant dans un plaisir illusoire,

17 Sénèque, Epistulae 108. 6. Cf. Pline le Jeune, Epistulae 1. 13; 5. 17; 6.17; 9. 34.

${ }^{18}$ Juvénal, Satires 6. 349-359. Pour Eppia, cf. ibid. 82-113 («une simple femme» dit Juvénal); pour Laevina, Martial, Epigrammes 1. 62; pour Vistilia, Tacite, Annales 2. 85, 1-2.

19 Pétrone, Satiricon 111 sqq.

${ }^{20}$ Plaute, Miles gloriosus v. 698-700. 
l'homme romain s'est condamné à l'incapacité de trouver son bonheur. Certes, on a bien remarqué la solitude du héros Enée, vibrante émanation de l'âme romaine, lorsqu'il doit porter seul Anchise pour fuir Troie en flammes, lorsqu'il guide le navire, ou doit descendre aux Enfers, mais Enée est un héros, et l'humble mortel n'a pas la même envergure devant les épreuves de l'existence. C'est pourquoi les religions à mystères, venues d'Orient, qui promettent une vie heureuse dans l'au-delà au prix d'une initiation et d'une ascèse, connaissent un réel succès: elles offrent, au propre et au figuré, la voie du salut. Car le salut de l'âme devient la grande affaire de l'humanité, avec pour guide, des prêtres qui ne sont plus des administrateurs mais des directeurs de conscience. La religion n'est plus devoir civique, mais expression d'une foi personnelle.

La philosophie, à laquelle a recours une élite, présente bien des points communs avec ces religions orientales. Le maître à penser, comme Sénèque pour Lucilius, y joue le rôle du prêtre - directeur de conscience. Et Sénèque lui-même n'a pas honte d'avouer à son disciple qu'il suit, lui aussi, les leçons d'un autre philosophe, Metronax, à soixante ans sonnés ${ }^{21}$. Au reste, il emploie de nombreuses formules qui évoquent le sibi uiuere, mais qui le situent dans une perspective de réflexion sur soimême. L'homme doit se prendre pour objet d'étude puisque «Dieu t'a donné à toi-même», comme le dit Epictète. Et encore doit-il le faire sans tarder, ainsi que le lui rappelle Marc Aurèle: «viens-toi en aide si tu te souviens de toi-même, tant que c'est encore possible ${ }^{22}$.» Mais chaque citoyen n'a pas la force d'âme de faire son examen de conscience quotidien, comme le prônait Pythagore, ou de régler sa pensée sur ses actes, comme le conseille Epictète, de faire, selon le mot de Marc Aurèle, retraite «dans sa propre âme». Ce sont là des pratique d'un otium raffiné dont seuls des hommes de la qualité d'un Pline le Jeune sont capables.

Ce «souci de soi» prend donc une nouvelle signification à l'époque de Martial, et se double, dès le deuxième siècle, d'un autre soin complémentaire, celui du corps. Cet intérêt conjoint pour le salut de l'âme et la santé du corps va induire une nouvelle morale qu'accompagne un progressif «assagissement» des mœurs ${ }^{23}$. Tacite, entre autres, a bien compris

${ }^{21}$ Sénèque, Epistulae 76. 3.

22 Epictète, Entretiens 2. 8. 23. Marc Aurèle, Pensées 3. $14 ; 4.3$.

${ }^{23} \mathrm{Cf}$. notre Eros romain. Sexe et morale dans l'ancienne Rome (Paris, Les Belles Lettres, 1997), $4^{\mathrm{ème}}$ époque, pour l'analyse de cette évolution. 
les causes de cette évolution morale. Comme les philosophes avant lui, il constate les ravages occasionnés par la passion et le luxe dont les nobles les plus fortunés étaient devenus les esclaves: «plus on se faisait remarquer par ses ressources, sa demeure, son train de vie, plus, grâce à son nom et à sa clientèle, on devenait illustre ${ }^{24}$.» Mais deux facteurs confortent l'évolution constatée: d'une part les massacres et les affrontements pour le pouvoir, et bientôt la poussée des barbares aux frontières qui menacent la Pax romana - la peur invite à la modération, d'autre part l'arrivée à Rome aux postes les plus élevés d'hommes nouveaux venus des colonies et des provinces qui apportent avec eux l'économie héritée de leur éducation. Le renouvellement de la classe dirigeante change peu à peu les mœurs d'une aristocratie qui périt de ses propres folies. Les empereurs ne sont plus nécessairement d'origine romaine, ni même italienne; avec les Antonins, leur choix se fait par l'adoption fondée sur le mérite. Les femmes de la haute sociéte donnent parfois elles aussi l'exemple de la parcimonie, comme Plotine dont la simplicité et la modestie, vantées par Pline ${ }^{25}$, ont servi la gloire de Trajan.

A l'époque de Martial, la mentalité connaît une transformation en profondeur qui tient compte de tous ces facteurs et favorise la mise en place d'une morale nouvelle en harmonie avec la nouvelle quête spirituelle. Il s'agit d'une morale familiale, qui n'a que peu de rapports avec celle de la virilité héritée de l'héroïque époque républicaine, et qui se justifie par la double évolution des statuts de l'homme et de la femme dans la société. Le citoyen est devenu sujet, la femme jouit d'une considération nouvelle: on peut dire en quelque sorte que leur relation s'équilibre pour donner naissance à ce que Paul Veyne appelle «le mythe de l'amour conjugal». Deux notions sont à retenir dans cette formule, celle d'amour, qui fait référence à un sentiment moderne inconnu jusqu'alors dans cette signification, et celle de couple, également nouvelle à Rome avec sa référence à une morale conjugale bien différente de celle des citoyens de la République. Pour aller vite, cette nouvelle relation entre les époux sous-entend la fidélité de chacun des deux à l'autre, la condamnation de l'homosexualité et l'évolution des sentiments parentaux. La lecture des Epigrammes de Martial montre que la mutation fait

\footnotetext{
${ }^{24}$ Cf. Tacite, Annales 3. 55.

${ }^{25}$ Pline le Jeune, Panégyrique de Trajan 84.
} 
son chemin dans les esprits, mais n'est pas encore accomplie, notamment dans la mentalité populaire. Il y demeure encore un vieux fond de «bisexualité de viol» (selon le mot de P. Veyne) héritée de la République ${ }^{26}$. Néanmoins, $c^{\prime}$ est bien à l'édification de la nouvelle morale païenne que nous assistons, celle sur laquelle s'appuieront les chrétiens pour imposer les règles morales dites judéo-chrétiennes qui sont censées être toujours les nôtres aujourd'hui.

Trois images suffisent à symboliser trois moments de cette évolution des sentiments amoureux: la première est celle du censeur Caton qui raye de l'album un sénateur qui a osé embrasser sa femme en présence de leur fille ${ }^{27}$. Un sentiment ne se montre pas; il est dangereux d'y céder; un citoyen doit savoir se maîtriser, c'est-à-dire rester le maître. La deuxième est celle de l'amour qui ose s'avouer, celui de Catulle par exemple, mais il ne s'agit que de passion, au demeurant tout à fait répréhensible ${ }^{28}$ (odi et amo). La troisième se résume à cette lettre que Pline le Jeune écrit à sa femme, absente momentanément: "vous ne sauriez croire combien vous me manquez. La raison en est mon amour, d'abord, puisque nous n'avons pas l'habitude d'être éloignés l'un de $\mathrm{l}^{\prime}$ autre ${ }^{29} \ldots$ » Trois images qui illustrent l'étonnante évolution des relations entre les êtres.

L'analyse des sentiments est même poussée par les philosophes jusqu'au raffinement, comme le montre bien Apulée dans son De Platorie. L'auteur y distingue trois degrés de l'amour. Aux deux extrémités se situent, d'une part l'amour des «âmes sombres», l'amour terrestre, éprouvé par ceux qui apaisent leur ardeur dans la jouissance du plaisir des sens, et d'autre part «l'amour divin», «puisque proche de la raison», et inspiré «d'un désir céleste». Entre les deux, il existe une sorte d'état intermédiaire, "résultant de la juxtaposition» des deux autres formes d'amour, touchant «les âmes moyennes» qui «ne sont pas complètement exemptes des plaisirs procurés par le corps», mais qui "peuvent être

${ }^{26}$ Paul VEYNE, «La famille et l'amour sous le Haut-Empire romain», in Annales ESC n¹, 1978.

27 Plutarque, Cato Maior 17. 7.

${ }^{28}$ Catulle, Carmina 85.

${ }^{29}$ Pline le Jeune, Epistulae 7. 5. 
séduites par le charme naturel des âmes ${ }^{30} »$. Or il est possible d'appliquer ces considérations comme grille de lecture des Métamorphoses, la dernière œuvre d'Apulée. Le héros, Lucius, avide du plaisir physique pour l'accorte Photis, n'est qu'une «âme sombre». Justement transformé en âne, il pourra assister en témoin à de nombreux autres exemples de cet amour terrestre. Mais, lorsque délivré par Isis de son apparence animale, il promet de se consacrer à la déesse avec «les difficiles obligations de chasteté et d'abstinence», il touche à "l'amour céleste»; Isis lui promet le bonheur sous sa protection, à son service et dans l'au-delà: «si ta dévotion est sans faille,... tu sauras que j'ai, moi seule, le pouvoir de prolonger ta vie au-delà des limites fixées par ton destin.» C'est, pour Lucius, «un bonheur ineffable», comme une «naissance mystique», et l'abstinence est la meilleure garantie d'abstraction du corps au bénéfice de l'esprit.

Quant à l'amour intermédiaire, celui qui mêle les plaisirs du corps à l'épanouissement de l'âme, il est illustré par l'apologue d'Eros et Psyché, au cœur même du roman. Eros n'avait connu que l'amour terrestre. Avec Psyché et la naissance de leur fille, ils découvrent ensemble cet amour intermédiaire, cette «juxtaposition de l'amour divin et de l'amour terrestre» dont parle de De Platone, bien exprimé par le nom même de l'enfant, Voluptas et celui de sa mère, Psyché. On peut remarquer d'ailleurs, qu'à l'occasion de cette naissance, la jeune femme, qui symbolise l'existence de la femme romaine, passant de l'autorité paternelle à celle de son époux et, au départ, traitée par celui-ci comme une enfant, devient peu à peu pour Eros une partenaire. Et c'est dans cet équilibre qu'ils éprouvent ensemble un amour réciproque.

$* * * * * * * * * *$

L'époque de Martial correspond à une période de crise politique et morale. Il faut au pouvoir impérial prendre la mesure d'un aussi vaste empire et lui assurer paix et équilibre. Un bon siècle sera encore nécessaire pour que tout homme libre devienne un citoyen à égalité avec les fils de Romulus. Mais la crise, inévitable, fut salutaire parce qu'elle a fait prendre conscience de l'obligation d'évoluer et de concevoir un cadre moral si fédérateur que la religion dominante, à la fin de l'Empire, se contentera de l'habiller de son propre symbolisme. Rome n'était sans

${ }^{30}$ Apulée, De Platone 2. 14. 
doute plus dans Rome, puisqu'elle était partout, mais elle avait réussi cette périlleuse mutation là où tant d'autres avaient échoué.

Notre vision du monde est, à l'évidence, très différente de celle des Romains, et l'étude de leur mentalité, c'est-à-dire du regard porté par eux sur le monde et les hommes, montre tout le chemin que nous avons parcouru. Mais la civilisation romaine peut nourrir notre réflexion sur nous-mêmes. Rome n'est pas un modèle, encore moins le modèle de notre mentalité, mais elle reste une expérience unique et privilégiée.

Unique, parce que la langue et l'esprit latins sont le dénominateur commun des traditions juive et chrétienne d'une part, et païenne d'autre part qui forment les deux éléments irréductibles l'un à l'autre dans la réalisation de l'unité culturelle de l'Europe. Les Romains ont assuré cette transmission, non point en serviles copieurs comme l'ont dit certains, mais en auteurs d'une géniale latina interpretatio, en médiateurs et pacificateurs qui ont procédé, selon le mot de Péguy, à «une greffe unique au monde.» Et ils ont du même coup assuré à la pensée grecque une postérité qu'elle n'aurait pas connue sans eux.

Expérience privilégiée également parce que Rome est le berceau de cette latinité qui définit notre sentiment actuel d'appartenir à une même civilisation européenne, et aussi parce qu'elle propose une vision unique et remarquable de l'homme et une tentative exemplaire de le considérer, de le fédérer à son projet universel en le respectant. En un mot, l'expérience d'un humanisme qui mérite qu'on s'y arrête parce que l'homme y fut traité avec ses faiblesses, certes, mais encore dans sa grandeur. Par sa quête de la paix et de la concorde civile, la concordia a forgé un modèle d'esprit communautaire et le principe de la cohésion sociale. L'homme s'efface alors devant le monde, sa nouvelle patrie: cum hac persuasione uiuendum est: non sum uni angulo natus, patria mea totus hic mundus est, proclame Sénèque ${ }^{31}$. Tel est bien l'humanisme romain, qui touche à l'universel.

${ }^{31}$ Sénèque, Epistulae 28. 4. 